\title{
Forbush decrease of June 8, 1969: Causes of the unusually long recovery
}

\author{
S. O. Ifedili \\ Department of Physics, University of Benin, Benin City, Nigeria \\ (Received August 16, 2000; Revised May 28, 2001; Accepted June 13, 2001)
}

\begin{abstract}
The cosmic-ray decreases and interplanetary disturbances, that occurred at 1 AU during the period, June 8-July 21,1969 , have been investigated by using the cosmic-ray intensity data recorded with ground-based monitors at Mt. Washington and Deep River, as well as the Interplanetary Magnetic Field (magnitude and direction) and the Solar Wind Plasma bulk speed, density and temperature at 1 AU. We observed a two-step Forbush decrease on June $8-9,1969$, which was due to the structure within the shock and sheath preceding the interplanetary coronal mass ejection. We also observed two cosmic-ray depressions on June 16-20 and July 13-15, 1969, which were attributed to a long-lived corotating high-speed solar wind stream. The outward propagating interplanetary shock waves that occurred at about 0700 UT on June 16, 1969, and at about 1400 UT on July 13, 1969 and which were associated with the long-lived corotating high-speed solar wind stream, most probably swept away the galactic cosmic rays, causing the delay in the Forbush decrease recovery at $1 \mathrm{AU}$, and, hence, the unusually long recovery of the two-step Forbush decrease at 1 AU with onset on June 8, 1969. The additional depressions by the interplanetary shocks associated with the long-lived corotating high-speed solar wind streams which were superposed on the recovery phase of the Forbush decrease of June 8, 1969, were shown to be larger on the lower energy galactic cosmic-ray particles, and therefore the duration of the recovery phase would be much longer in the lower energy region, an expectation which is consistent with the observations.
\end{abstract}

\section{Introduction}

Forbush decreases (FDs) are transient and rapid (typically within a few hours to a few days) decreases (FDs) of the cosmic-ray intensity; the decreases are followed by a slow recovery typically lasting several days. Though FDs are believed to be produced by perturbations in the interplanetary conditions, none of the theories (e.g., Gold, 1960; Parker, 1963; Barnden, 1973; Nishida, 1983; Kadokura and Nishida, 1986) so far advanced to explain FDs, has succeeded in completely explaining the details of this phenomenon. The perturbations could be produced by shock waves, moving magnetic clouds or high-velocity solar wind streams (e.g., Lockwood, 1971; Burlaga et al., 1981; Zhang and Burlaga, 1988; Lockwood et al., 1991; Fluckiger, 1991; Cane et al., 1994; Cane and Richardson, 1995; Ifedili, 1996a, 1997).

This paper investigates one of the most famous FDs in galactic cosmic-ray intensity, which occurred on June 8, 1969 close to the sunspot maximum of solar cycle 20 . The event is well known by its abnormally slow recovery after the maximum intensity depression. The recovery from the decrease in this event took as long as several months even in high-energy region covered by the ground based neutron monitors, while it takes less than a week in most of usual events. An important feature of this event is that the duration of the recovery phase was much longer in lower energy region than in high-energy region. This feature clearly distin-

Copy right (c) The Society of Geomagnetism and Earth, Planetary and Space Sciences (SGEPSS); The Seismological Society of Japan; The Volcanological Society of Japan; The Geodetic Society of Japan; The Japanese Society for Planetary Sciences. guishes this event from others in the course of 11 year modulation of galactic cosmic ray intensity. In order to understand this feature in the framework of the quasi-stationary model for the galactic cosmic ray modulation, some researchers needed to introduce abrupt change in either energy dependence of the modulation function (Lockwood et al., 1972) or distribution of solar activity on the solar disk (Iucci et al., 1974).

This paper proposes an alternative and new idea to understand the unusually long recovery phase of the FD in terms of the recurring intensity depressions due to the long-lived corotating solar wind streams (CSWSs) superposed on the recovery phase of the FD. The typical signatures of CSWSs are found directly in the solar wind plasma data during the recovery phase. This alternative idea naturally explains the long recovery of the FD, as well as the observation that the duration of the recovery phase was much longer in the lower energy region than in high energy region.

\section{The Forbush Decreases, and Interplanetary Disturbances}

Figure 1 displays the daily average cosmic-ray intensity from June 1 through July 31, 1969 as recorded by Mt. Washington (Fig. 1(a)) and Deep River (Fig. 1(b)) ground-based detectors. The counting rates have been normalized to $100 \%$ on June 7, 1969. The figure indicates that the amplitude of the Forbush decrease, June 8-10, 1969 at Mt. Washington and Deep River is $4.6 \%$ and $3.9 \%$, respectively, which is about a fifth of the complete solar cycle variations. Here, Forbush decrease amplitude (\%) is calculated as $100\left(N_{o}-\right.$ $\left.N_{F}\right) / N_{o}$, where $N_{o}$ is the daily counting rate of the detector 


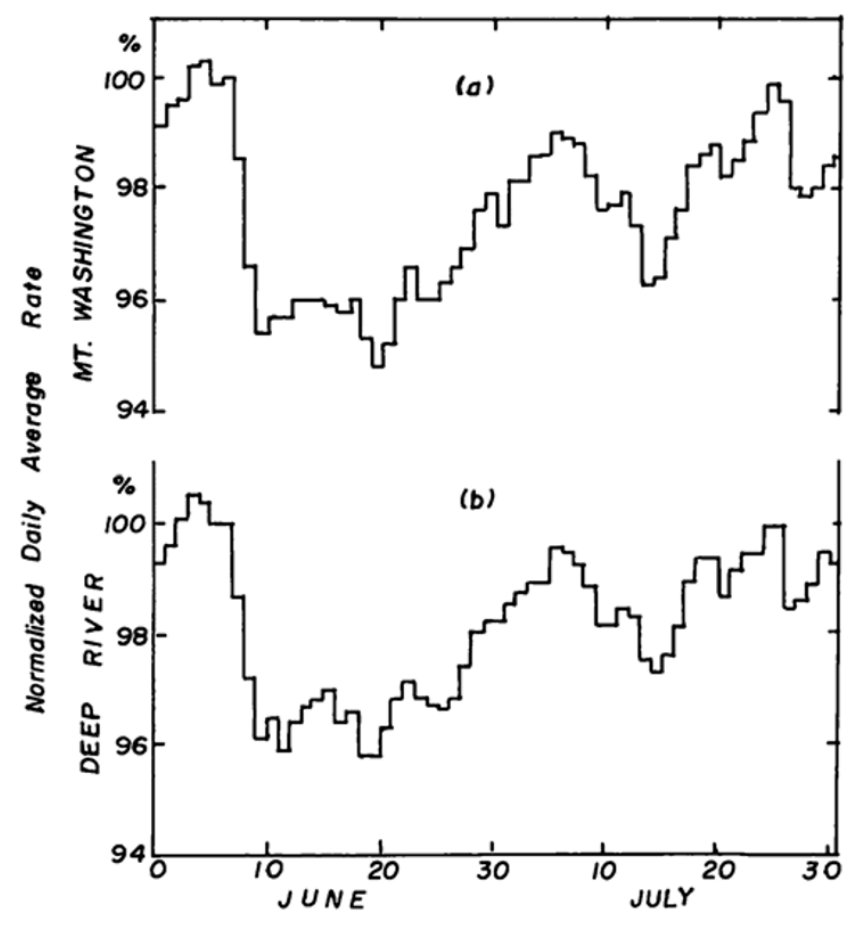

Fig. 1. Daily average counting rates of the neutron monitors at (a) Mt Washington $\left(P_{c}=1.24 \mathrm{GV}\right)$ and (b) Deep River $\left(P_{c}=1.02 \mathrm{GV}\right)$ during the period, 1969 June $1-$ July 31 . The counting rates have been normalized to $100 \%$ on June $7,1969$.

before the decrease and $N_{F}$ the daily rate during the maximum depression of the cosmic-ray rates.

Notice the unusually long recovery phase of the Forbush decrease of June 8-10, 1969, at the Earth in Fig. 1. Observation of a high depression of the cosmic-ray particles at low cut-off rigidities $(<0.6 \mathrm{GV})$ during the recovery phase has been previously reported (e.g., Lockwood, 1971; Lockwood et al., 1972; Ifedili, 1973; Iucci et al., 1974; Ifedili, 1996b). However, no attempts have been made to identify the interplanetary conditions that caused the unusually long recovery of the FD that occurred at 1 AU on June 8, 1969.

Figure 2 shows the 3-hour averages of the cosmic-ray intensity at Deep River as well as of the Interplanetary Magnetic Field (magnitude and direction) and the Solar Wind Plasma (SWP) data at 1 AU from the National Space Science Data Center (King, 1977a) during the periods, June 8-26 and July 10-21, 1969 during which the major cosmicray intensity depressions occurred. The depressions on June 12 and June 25-26 are enhanced diurnal variations because each of the depressions has a periodicity of $\sim 24$ hours and was not observed at similar times at some other neutron monitors such as Alert neutron monitor.

A Storm Sudden Commencement (SSC) which occurred at 0500UT on June 8, 1969 marked the arrival of an interplanetary shock wave at the Earth; the shock arrival time coincided with a sudden increase of both the Interplanetary Magnetic Field (IMF) intensity and the SWP bulk speed, and preceded (by about 1 hour) the onset time of the FD of about $2.9 \%$ in amplitude at Deep River, as can be seen from Fig. 2. Thenceforth, the IMF intensity took on a very significant role: a rapid decrease ( $\geq 1 \mathrm{nT} / \mathrm{hr}$ ) during the periods (a) about 0500 UT to about 1600 UT on June 8 and

(b) about 2100 UT on June 8 to about 0500 UT on June 9; and

a lull ( $\precsim 0.3 \mathrm{nT} / \mathrm{hr}$ ) during the interval, about 1600 UT to about 2100 UT on June 8.

While this rapid decrease of the IMF intensity coincided with the depressions in the cosmic ray intensity, i.e. the onset phases of the first and second steps of the Forbush decrease, the lull coincided with the recovery phase of the first step, an indication that the large variations in IMF sustained the depressions in the cosmic ray intensity. The cosmic ray intensity was also depressed during the period of the IMF rotation and elevated intensity, at about 0500 UT on June 9 to about 1100 UT on June 10, 1969. The amplitude of the second decrease was about $2.1 \%$.

These observations are not consistent with the model of Barnden (1973) which interprets the two-step FD as caused by the post-shock turbulence followed by entry into the shock driver or ejecta, since the second decrease started before the entry into the magnetic cloud.

The two-step FD was due to the structure within the shock and sheath preceding an interplanetary coronal mass ejection (ICME). ICME is the solar CME (coronal mass ejection) that is able to get out into interplanetary space. There is a sheath upstream of the ICME led by a fast forward shock as is recognised in Fig. 2 where the first vertical dashed line indicates the approximate time of arrival of the fast forward shock at $1 \mathrm{AU}$. The sheath material is slow solar wind that has been swept up by the ICME, and is not part of the ICME itself. Figure 2 shows the sheath region is sufficiently turbulent as indicated by the large variations in both the field strength and direction in the region. The sheath has a high speed of about $400 \mathrm{~km} / \mathrm{s}$, which is much larger than the ambient speed of about $300 \mathrm{~km} / \mathrm{s}$, was most probably produced by the shock, and is conceivably hot $\left(\succsim 10^{5} \mathrm{~K}\right)$ and dense $\left(\succsim 10 \mathrm{~cm}^{-3}\right)$ though there is a data gap in solar wind density and temperature in the region. Therefore, the large IMF variations in the shocked plasma scatter the galactic cosmic rays, and thus sweep away the cosmic-ray particles. When the IMF variations in the sheath become too feeble, i.e. in the lull region, the scattering of the galactic cosmic rays ceases; the scattering resumes, i.e. onset of second decrease, when the IMF variations in the sheath resume. The second region is the ICME/ejecta (magnetic cloud) which is the region between the two vertical solid lines in Fig. 2. This high field region could sweep out cosmic-ray particles as well. This region is labelled MAGNETIC CLOUD since the IMF data show a high field strength and rotation signature of the magnetic cloud; the magnitude of the magnetic field is up to about $10 \mathrm{nT}$, which is much larger than the ambient field of about $5 \mathrm{nT}$. In magnetic clouds, the field strength is high and the density and temperature are relatively low (e.g., Burlaga et al., 1981; Zhang and Burlaga, 1988; Ifedili, 1999). The total pressure inside the cloud is higher than outside, implying that the cloud is expanding as it moves outward (Burlaga et al., 1981). Because of the expansion of the magnetic cloud as it moves outward from the solar corona, near Earth the temperature and density in the 


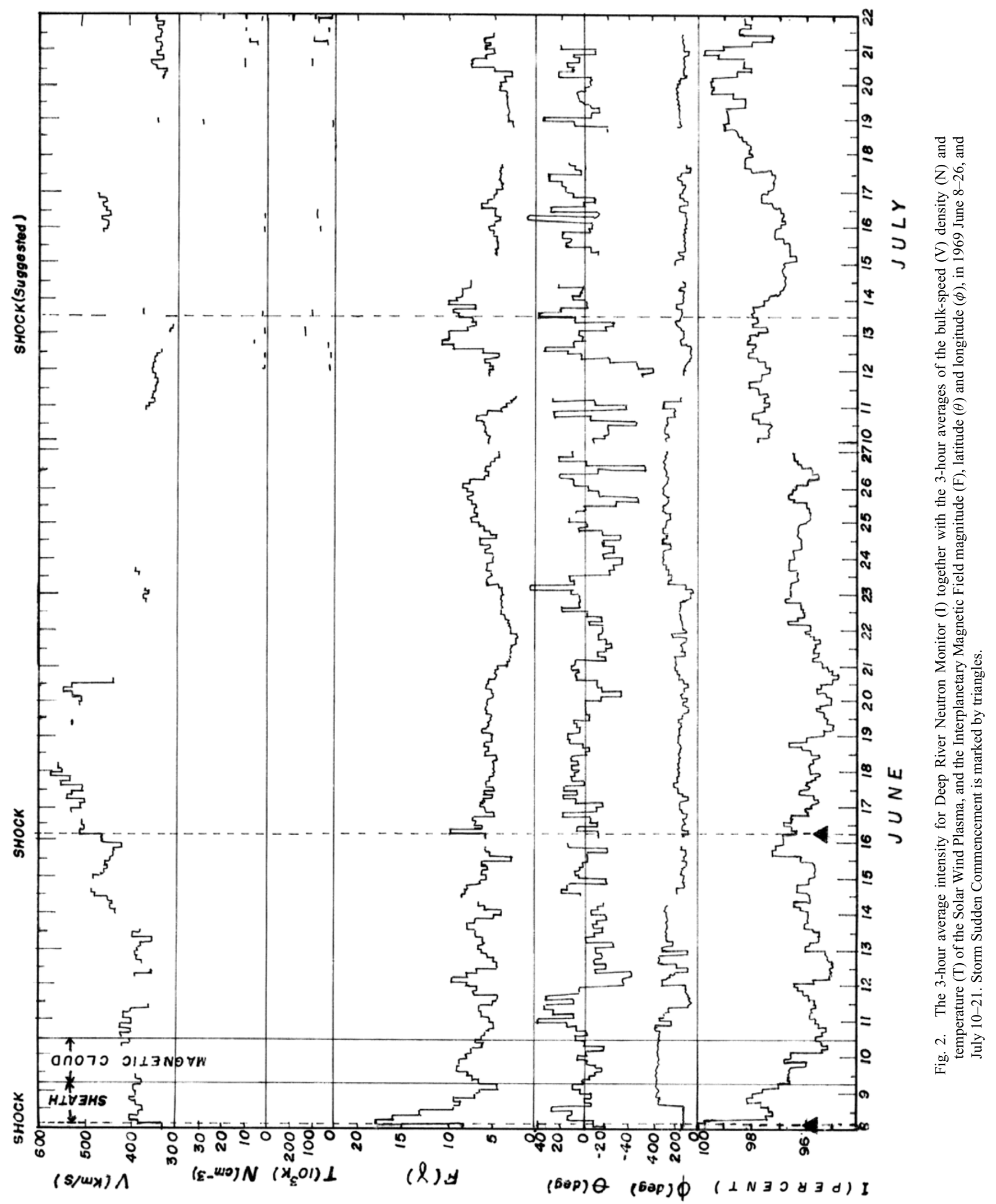




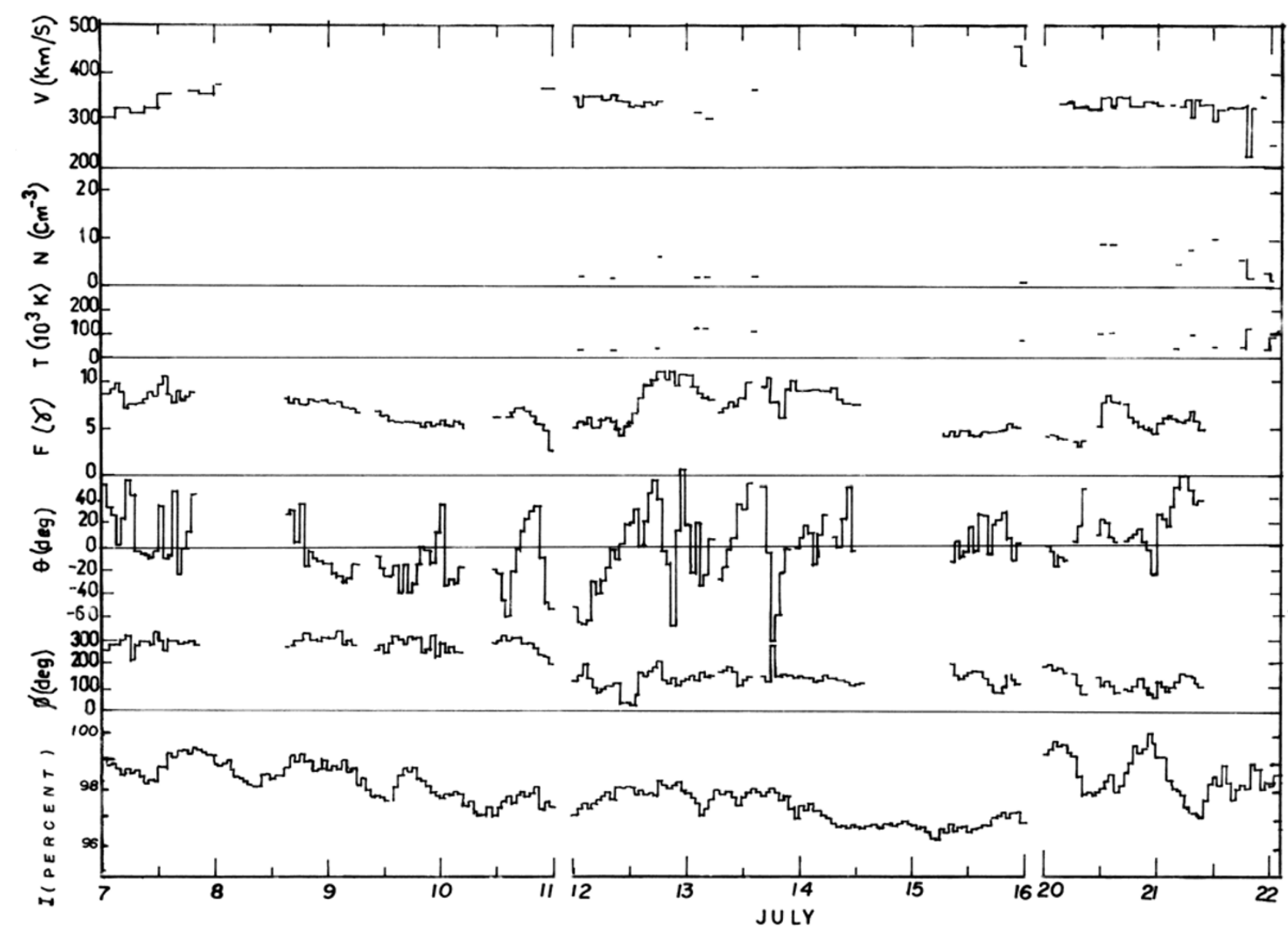

Fig. 3. The hourly average intensity for Deep River Neutron Monitor (I) together with the hourly averages of the bulk-speed (V), density (N) and temperature (T) of the Solar Wind Plasma, and the Interplanetary Magnetic Field magnitude (F), latitude $(\theta)$ and longitude $(\phi)$ in 1969, July 7-10, $12-15$, and $20-21$.

cloud would have reduced to the low enough values, $\precsim 10^{4} \mathrm{~K}$ and $\precsim 1 \mathrm{~cm}^{-3}$, respectively. When the expanding magnetic cloud envelopes the Earth, some of the galactic cosmic rays are scattered away from the high magnetic field pressure in the cloud; and therefore the cosmic ray intensity within the magnetic cloud is temporarily depressed below that in the surrounding regions.

Another SSC which occurred at about 0700 UT on June 16, 1969 marked the arrival of an interplanetary shock wave at the Earth; the shock arrival time coincided with a sudden increase of both the IMF intensity and the SWP bulk speed, and preceded by about 9 hours the onset time of the cosmic-ray depression of amplitude about $2.0 \%$ at Deep River; the maximum depression occurred at about $1500 \mathrm{UT}$ on June 20, 1969, and the decrease was slow and continuous, as shown in Fig. 2.

The interplanetary data in Fig. 2 suggest that the decrease on June 16-20 was caused by a CSWS. The magnitude of the magnetic field is enhanced, and the plasma speed rises gradually at the leading edge of the stream, and then, the SWP speed falls, while the magnetic field azimuth remains constant (away from the Sun); the solar wind speed and cosmic-ray intensity are approximately anti-correlated, as is typical in a corotating stream (e.g. Richardson et al., 1996). A similar stream was observed (King, 1977b), 1 solar ro- tation earlier (from May 21, 1969), and a rotation later, from 13-15 July 1969, as is also evident in Fig. 2, providing further evidence that this was a CSWS. Note that the respective maximum percentage increases of about $29 \%$ and $27 \%$ in SWP speed during its gradual rise on June 16-20, and on July $13-15$ are approximately coincident with the respective maximum percentage decreases of about $2.0 \%$ and $1.7 \%$ in the cosmic-ray intensity. Notice also, in Fig. 2, that during the period of the cosmic-ray intensity decrease on June 1620 , the IMF intensity was near its background level of about $5 \mathrm{nT}$, except at the leading edge of the stream where the IMF intensity increased to about $10 \mathrm{nT}$. It appears, therefore, that the cosmic-ray intensity decrease on June 16-20 was caused by the interplanetary shock wave that was associated with high-speed solar wind plasma. Notice the vertical dashed line in Fig. 2 at about 1400 UT on July 13, 1969 when the IMF intensity at $1 \mathrm{AU}$ increased to about $9.5 \mathrm{nT}$, and (where data are available) the SWP speed at 1 AU was enhanced and the SWP density and temperature at $1 \mathrm{AU}$ were higher than their background values, suggesting the presence of a shock wave. It took about 7 and $9+$ days respectively, for the June 16-20 and July 13-15 cosmic-ray depressions, to complete the onset and recovery phases.

For a more detailed study, the data in Figs. 1 and 2 have been expanded to a much higher resolution of 1 hour in 
Fig. 3 for the periods, July $7-10,12-15$ and $20-21$. The depressions on July 7-10, and 20-21, are most probably due to enhanced diurnal variation, since each of the depressions has a periodicity of $\sim 24$ hours and was not observed at similar times at some other neutron monitors such as Alert neutron monitor.

There is no evidence in Figs. 2 and 3 of the presence of a typical magnetic cloud during the recovery phase of the FD of June 8,1969 . The recovery phase began at about 1100 UT on June 10. Figure 2 shows that on June 26, 1969, the IMF changed direction continuously from $48^{\circ} \mathrm{N}$ at $0800 \mathrm{UT}$ to $74^{\circ} \mathrm{S}$ at $1400 \mathrm{UT}$, and then to $48^{\circ} \mathrm{N}$ at $1900 \mathrm{UT}$, but the field rotation is probably not due to a typical magnetic cloud because the IMF intensity was depressed during the field rotation on June 26 . The field rotation from $60^{\circ} \mathrm{S}$ at 1500 $\mathrm{UT}$ to $31^{\circ} \mathrm{N}$ at $2100 \mathrm{UT}$, and then to $53^{\circ} \mathrm{S}$ at $2400 \mathrm{UT}$, on July 10, 1969 in Fig. 3, was probably not a typical magnetic cloud because the IMF intensity was depressed. The field rotations from $53^{\circ} \mathrm{N}$ at $1800 \mathrm{UT}$ to $68^{\circ} \mathrm{S}$ at $2200 \mathrm{UT}$, and then to $63^{\circ} \mathrm{N}$ at 2400 UT on July 12 , were not magnetic clouds because the SWP density $6.3 \mathrm{~cm}^{-3}$, was too high for a typical magnetic cloud. Neither was the field rotation from $50^{\circ} \mathrm{N}$ at $1700 \mathrm{UT}$ to $83^{\circ} \mathrm{S}$ at $1900 \mathrm{UT}$ on July 13, a typical magnetic cloud because the IMF intensity was depressed.

Thus, the outward propagating interplanetary shock waves which were associated with a CSWS most probably swept the galactic cosmic-rays ahead of them, to cause the two cosmic-ray depressions of June 16-20 and July 13-15, and delayed the recovery of the two-step Forbush decrease of June 8, 1969 for about 16+ days.

Therefore, whenever additional depressions by interplanetary shocks associated with two or more CSWSs, are superposed on the recovery phase of a Forbush decrease, unusually long recovery would result. However, this situation is expected to be rare on Earth, since for most Forbush decrease events on Earth, the recovery would have been completed before the passage of the second CSWS, which, therefore, would be unable to add to the contributions of the first that passed, 1 solar rotation $(\approx 27$ days) earlier. If only one CSWS contributes, then, the FD recovery, though proportionately lengthened, would still be too short to be unusual; CSWSs are not unusual structure but almost always exist in interplanetary space.

\section{Dependence of Length of Recovery on Energy}

Ifedili (1996a) used the cosmic-ray observations on Mt. Washington ground-based neutron monitor and on the 0G06 and Pioneer 8 spacecraft, to show that the depressions in the cosmic ray intensity at $1 \mathrm{AU}$, caused by the interplanetary shock waves and/or CSWSs, were larger at lower cutoff rigidities and at higher geomagnetic latitudes. The spacecraft data near Earth showed that, for vertical cut-off rigidities $P_{c} \leq 1.8 \mathrm{GV}$, the total percentage decrease in the amplitudes of the cosmic ray depressions can be represented by $-m P_{c}+k$, where $m$ and $k$ are each constant for the particular depression "but which increase with increasing Mt. Washington neutron monitor monthly average rates, an indication of a flattening of the rigidity dependence of the depressions towards maximum solar modulation." In particular, for the FD of June 8, 1969, $m$ and $k$ were respectively $2.4 \%$ per
GV and 5.8\%, giving the total percentage decreases of 5.2\% at $P_{c}=0.30 \mathrm{GV}, 3.35 \%$ at $P_{c}=1.02 \mathrm{GV}$, and $2.8 \%$ at $P_{c}=1.24 \mathrm{GV}$, which shows that the lower energy cosmicray charged particles were much more depressed than the high-energy particles.

Thus, the additional depressions by CSWSs associated with interplanetary shocks, which were superposed on the recovery phase of the FD of June 8, 1969, would be larger on the lower energy galactic cosmic-ray particles. For instance, using the daily average rates in Fig. 1 and for the additional depression of June 16-20, 1969, by CSWSs, where the amplitude of the depression at Mt. Washington $\left(P_{c}=1.24\right.$ $\mathrm{GV}$ ) was about $1.2 \%$, the amplitude at the lower cut-off $P_{c}=0.30 \mathrm{GV}$ would be about $2.2 \%$ according to the observed relation for the total percentage decrease in the amplitudes of the cosmic-ray depressions as given by Ifedili (1996a).

Conceivably, the larger the amplitude of the cosmic ray depression at $1 \mathrm{AU}$ due to a particular shock or CSWS, the longer the time to complete its onset and recovery phase. Thus, it is reasonable to assume a direct proportionality relationship between the amplitude and the duration of the depression. In this case, since it took about 7 days for the June 16-20, 1969 depression at Mt. Washington $\left(P_{c}=1.24 \mathrm{GV}\right)$ to complete the onset and recovery phases, the corresponding duration at $P_{c}=0.30 \mathrm{GV}$ and $P_{c}=1.02 \mathrm{GV}$ is about 12.8 and 8.4 days, respectively, which is a much longer duration than that at the higher cut-off $P_{c}=1.24 \mathrm{GV}$. However, a close inspection of Fig. 1 shows that the duration of about 7 days for the June 16-20, 1969, depression at Mt. Washington $\left(P_{c}=1.24 \mathrm{GV}\right)$, which has been used for the calculations, may have been underestimated; our estimates are conservative, and give reasonable values of length of recovery at different energies. Similarly, for the additional cosmicray depression of July 13-15, 1969, where the amplitude, and the duration of the onset and recovery phases, of the depression at Mt. Washington $\left(P_{c}=1.24 \mathrm{GV}\right)$ were respectively about $1.6 \%$ and 13 days, the corresponding duration at $P_{c}=0.30 \mathrm{GV}$ and $P_{c}=1.02 \mathrm{GV}$, and at this level of solar modulation, would be respectively about 24.1 and 15.6 days, which duration is again each much longer than that at $P_{c}=1.24 \mathrm{GV}$. Consequently, because of these two additional cosmic ray depressions by CSWSs associated with interplanetary shocks, the total delay in the recovery of the two-step FD of June 8, 1969 at $P_{c}=0.30 \mathrm{GV}$ and $P_{c}=1.02$ $\mathrm{GV}$, and at $1 \mathrm{AU}$, would be about 37 and 24 days, respectively, which is each much longer than the delay of about 20 days at the higher cut-off $P_{c}=1.24 \mathrm{GV}$. Thus the duration of the recovery phase of the FD of June 8, 1969 at $1 \mathrm{AU}$ would consequently be much longer in the lower energy region than in the high energy region, an expectation which is consistent with the following observations:

The approximate dates in 1969 when the FD of June 8 recovered to its predecrease level were:

(a) about July 25 at $\operatorname{Kula}\left(P_{c}=13.3 \mathrm{GV}\right)$ as obtained from figure 1 of Lockwood et al. (1972)

(b) August 13 at Mt. Washington $\left(P_{c}=1.24 \mathrm{GV}\right)$ as in Fig. 4 of the present paper. 


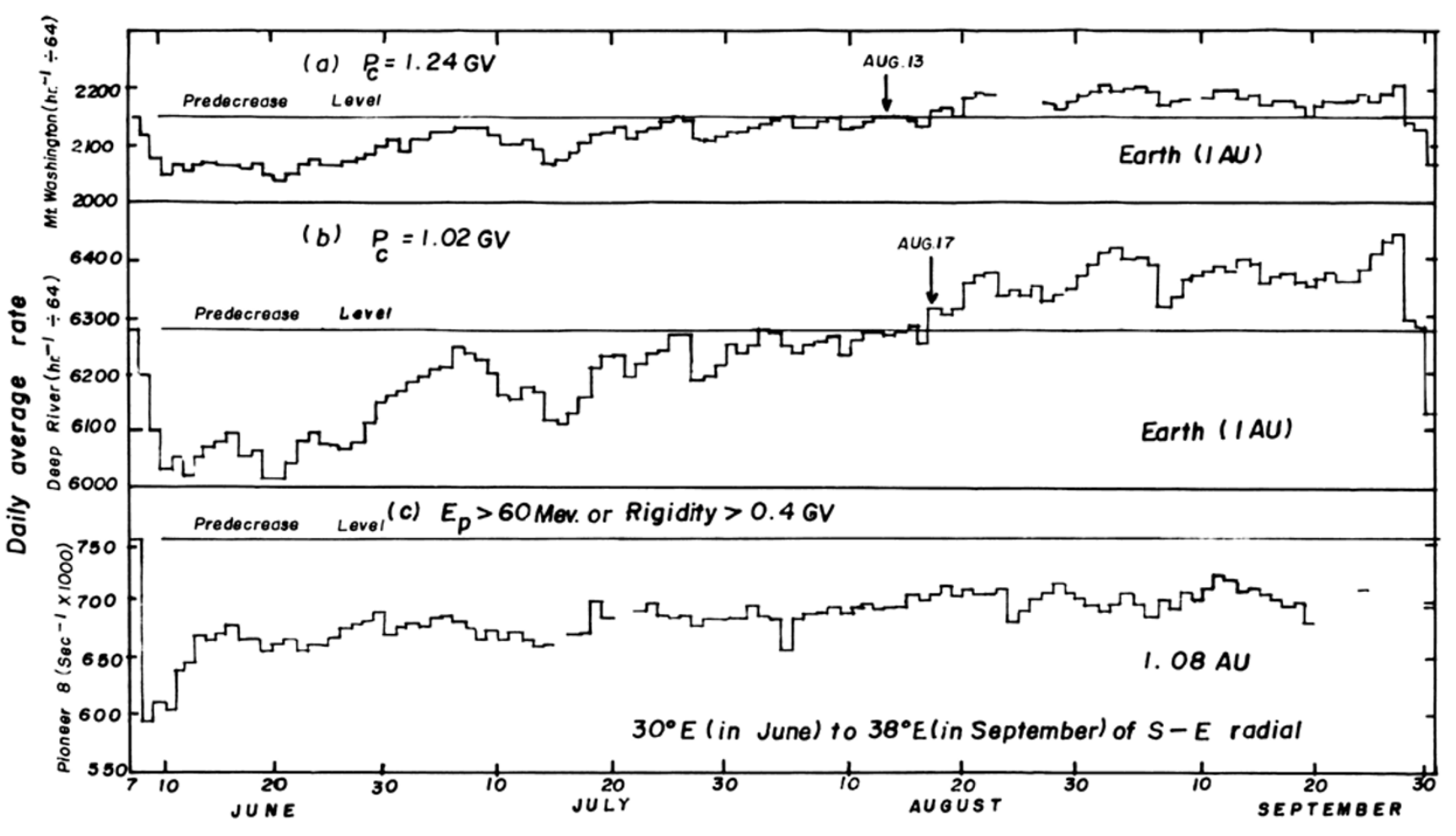

Fig. 4. Daily average counting rates in 1969 of the neutron monitors at (a) Mt. Washington $\left(P_{c}=1.24 \mathrm{GV}\right)$, (b) Deep River $\left(P_{c}=1.02 \mathrm{GV}\right)$, and of the cosmic-ray telescope $\left(E_{p}>60 \mathrm{MeV}\right.$ or rigidity $\left.>0.4 \mathrm{GV}\right)$ at (c) Pioneer 8 . The arrows indicate the dates when the cosmic-ray intensities returned to their predecrease levels.

(c) August 17 at Deep River $\left(P_{c}=1.02 \mathrm{GV}\right)$ as in Fig. 4 of the present paper.

Figure 4 shows the daily average rates of the cosmic ray intensity at Mt. Washington (Fig. 4(a)), Deep River (Fig. 4(b)) and Pioneer 8 telescope (Fig. 4(c)).

Thus, the duration of the recovery of the Forbush decrease of June 8, 1969 at $P_{c}=1.24 \mathrm{GV}$ was about 19 days longer than that at $P_{c}=13.3 \mathrm{GV}$, but about four days shorter than that at $P_{c}=1.02 \mathrm{GV}$, as expected.

However, the cosmic-ray intensity was still depressed in September 1969 at the Pioneer 8 cosmic ray telescope, even though there are data gaps in Fig. 4(c) during the periods, September 20 to 23, and 25-29. The telescope responds to protons with $E_{p}>60 \mathrm{Mev}$ or rigidity $>0.4 \mathrm{GV}$, and was located at about $1.08 \mathrm{AU}$ and $30^{\circ} \mathrm{E}$ (in June) to $38^{\circ} \mathrm{E}$ (in September) of the S-E radial. Thus, the spacecraft was evidently not near the Earth during the period, June to September 1969, and was then most probably not in the same modulating region as the Earth. Consequently, the cosmic-ray intensity observed in June to September, 1969, would not be as depressed at the Earth as at Pioneer 8.

The agreement between our expectations and the observations appears reasonable, considering the uncertainties especially in the estimates of the duration of the depressions, and in the possibly excluded residual time delay in the two months of data at solar maximum.

\section{Summary}

We observed a two-step FD on June 8-9, 1969, which was due to the structure within the shock and sheath preceding the interplanetary coronal mass ejection. We also observed two cosmic-ray depressions on June 16-20 and July 13-15, 1969, which were attributed to CSWS.

The interplanetary conditions for the FD of June 8, 1969 appear to indicate that the outward propagating interplanetary shock waves which were associated with the CSWSs, swept away the galactic cosmic-rays, causing the delay in the FD recovery at $1 \mathrm{AU}$, and, hence, the unusually long recovery of the two-step FD at $1 \mathrm{AU}$ with onset on June 8, 1969. The additional depressions by the interplanetary shocks associated with the CSWSs, which were superposed on the recovery phase of the FD of June 8, 1969, were shown to be larger on the lower energy galactic cosmic ray particles, and therefore the duration of the recovery phase would be much longer in the lower energy region than in the high energy region, an expectation which is consistent with the observations.

Acknowledgments. The author would like to thank Professor John Lockwood for his assistance with the neutron monitor data, Professor William Webber for providing the Pioneer 8 proton data, Professor J. H. King for providing the Interplanetary Magnetic Field and Solar Wind Plasma data, the referees for their very valuable comments, and the University of Benin, Nigeria, for a URPC grant.

\section{References}

Barnden, L. R., The Large-scale magnetic field configuration associated with Forbush decreases, Proc. 13th Int. Cosmic-Ray Conf. 2, 1277, 1973.

Burlaga, L. F., E. Sittler, F. Mariani, and R. Schwenn, Magnetic loop behind an interplanetary shock; Voyager, Helios, and IMP 8 observations, $J$. Geophys. Res., 86, 6673-6684, 1981.

Cane, H. V. and I. G. Richardson, Cosmic-Ray decreases and solar wind 
disturbances during late October 1989, J. Geophys. Res., 100, 1755, 1995.

Cane, H. V., I. G. Richardson, T. T. Von Rosenvinge, and G. Wibberenz, Cosmic-Ray decreases and shock structures: A multispacecraft study, $J$. Geophys. Res., 49, 21429, 1994.

Fluckiger, E. O., Solar and terrestrial modulation, Proc. 22nd Intern. Cosmic-Ray Conf., 5, 273-291, 1991.

Gold, T., Energetic particle fluxes in the solar system near the earth, Astrophys. J. Suppl. Ser., 4, 406, 1960.

Ifedili, S. O., Solar modulation of cosmic-ray albedo neutrons, Astrophys. Lett., 15, 49-54, 1973.

Ifedili, S. O., Spacecraft measurement of Forbush decreases in the cosmic radiation, Solar Phys., 168, 195-203, 1996a.

Ifedili, S. O., Forbush decreases in the cosmic-ray albedo neutron flux, $J$. Geomag. Geoelectr., 48, 1025-1031, 1996 b.

Ifedili, S. O., Forbush decreases in the cosmic radiation: Measurement of the extent of the modulating region, Planet. Space Sci., 45, 511-514, 1997.

Ifedili, S. O., Cosmic ray decreases and interplanetary disturbances during early November 1969, Planet. Space Sci., 47, 267-272, 1999.

Iucci, N., M. Parisi, and G. Villoresi. The change in the long-term modulation function of cosmic-rays in 1969, J. Geophys. Res., 79, 659-660, 1974.

Kadokura, A. and A. Nishida, Two dimensional modeling of the cosmic-ray storm, J. Geophys. Res., 91, 13-29, 1986.
King, J. H., Interplanetary medium Data Book-Appendix, NSSDC/WDCA-R \& S 77-04a, September, 1977a.

King, J. H., Interplanetary medium Data Book, NSSDC/WDC-A-R \& S7704, September, 1977b.

Lockwood, J. A., Forbush decreases in the cosmic radiation, Space Science Reviews, 12, 688-715, 1971.

Lockwood, J. A., J. A. Lezniak, and W. R. Webber, Change in the 11year modulation at the time of the June 8,1969 . Forbush decrease, $J$. Geophys. Res., 77, 4839-4844, 1972.

Lockwood, J. A., W. R. Webber, and H. Debrunner, The rigidity dependence of Forbush decreases observed at the earth, J. Geophys. Res., 96, 5447-5455, 1991.

Nishida, A., Numerical modeling of the energy spectrum of the cosmic ray Forbush decrease, J. Geophys. Res., 88, 785-791, 1983.

Parker, E. N., Interplanetary Dynamical Processes, Intersciences, John Wiley \& Sons, New York, 1963.

Richardson, I. G., G. Wibberenz, and H. V. Cane, The relationship between recurring cosmic ray depressions and corotating solar wind streams at 1 AU: IMP 8 and Helios 1 and 2 anticoincidence guard rate observations, J. Geophys. Res., 101, 13483, 1996.

Zhang, G. and L. F. Burlaga, Magnetic clouds, Geomagnetic disturbances, and cosmic ray decreases, J. Geophys. Res., 93, 2511-2518, 1988.

S. O. Ifedili (e-mail: ifedili@uniben.edu) 